\title{
Genome
}

\section{Comparative population genomics unveils candidate genes for athletic performance in Hanoverians}

\begin{tabular}{|c|c|}
\hline Journal: & Genome \\
\hline Manuscript ID & gen-2018-0151.R2 \\
\hline Manuscript Type: & Note \\
\hline $\begin{array}{l}\text { Date Submitted by the } \\
\text { Author: }\end{array}$ & 10-Feb-2019 \\
\hline Complete List of Authors: & $\begin{array}{l}\text { Asadollahpour Nanaei, Hojjat; Shahid Bahonar University of Kerman } \\
\text { Ayatollahi Mehrgardi, Ahmad; Shahid Bahonar University of Kerman } \\
\text { Esmailizadeh, Ali; Shahid Bahonar University of Kerman }\end{array}$ \\
\hline Keyword: & $\begin{array}{l}\text { whole genome analysis, athletic performance, Hanoverian horse, ACTA1 } \\
\text { gene }\end{array}$ \\
\hline $\begin{array}{r}\text { Is the invited manuscript for } \\
\text { consideration in a Special } \\
\text { Issue? : }\end{array}$ & Not applicable (regular submission) \\
\hline
\end{tabular}

\section{SCHOLARONE Manuscripts}


Comparative population genomics unveils candidate genes for athletic performance in Hanoverians

Hojjat Asadollahpour Nanaei ${ }^{1}$, Ahmad Ayatollahi Mehrgardi ${ }^{1}$, Ali Esmailizadeh ${ }^{1}$ *

1. Department of Animal Science, Faculty of Agriculture, Shahid Bahonar University of

Kerman, Kerman, PB 76169-133, Iran

${ }^{*}$ Corresponding author

Ali Esmailizadeh

aliesmaili@uk.ac.ir

ORCID ID: https://orcid.org/0000-0003-0986-6639

Tel: +98 34-33257443. Fax: +98 34-31322692 


\begin{abstract}
Equine athletes have a genetic heritage that has been evolved for millions of years, which provides an opportunity to study the genetics of locomotion pattern and performance in mammals. The Hanoverian, a breed originating in Germany, is arguably among the most athletic horse breeds, due to its balanced character and beautiful appearance. Here, we compared the whole genomes of Hanoverian with other three horse breeds (Akhal-Teke, Franches-Montagnes and Standardbred), using the fixation index (Fst) and cross-population composite likelihood ratio (XP-CLR) methods for testing the multilocus allele frequency differentiation between populations. We identified 299 and 485 positively selected genes using the Fst and XP-CLR methods, respectively. Further functional analyses showed that the ACTA1 gene is potentially involved in athletic performance in Hanoverian breed, consistent with its role observed in human population. In addition, three other loci on chromosomes 1 and 20 were identified to be potentially involved in the equine physical performance. The candidate selected genes identified in this study may be useful in current breeding efforts to develop improved breeds for the athletic performance.
\end{abstract}

Keywords: whole genome analysis; athletic performance; ACTA1 gene; Hanoverian horse 


\section{Introduction}

During the last decades, efforts to genetically improve the ability of sport competition has been growing in many horse breeders (Tomasz et al. 2015). Performance in show jumping is an important economic trait in the horse sport industry. Thus, many breeding organizations and majority of warmblood studbooks, have selection programs for such sport horses (Koenen et al. 2004). The Hanoverian (HNV), a horse breed originating in Germany, is arguably among the most athletic horse breeds, due to its balanced character and beautiful appearance. The history of the breed, like many other crossbreed German warmbloods, dates back from as early as 1735 , when some foreign stallions imported in the Celle stud. From then on, this breed has been successfully bred and developed to produce high standard level sport, and it is probably the world's largest warmblood horse today (Hamann and Distl 2008; Schröder et al. 2012). Recent technological developments in molecular biology made possible the large-scale sequencing data, which are essential for the interpretation of the animal and plant genomes (Zhang and Hewitt 2003; Leroy and Rowen 2013). One of the approaches for detecting candidate genes is screening for genomic regions that have been undergone natural or 
artificial selection (Yamasaki et al. 2005). Up to now, numerous candidate loci have been detected in experimental and commercial livestock species like cattle (Rothammer et al. 2013), sheep (Moradi et al. 2012) and pig (Wilkinson et al. 2013). Despite a late start, research in the field of equine genome has also progressed rapidly in recent years. For example, several linked markers have been reported for some traits of interest such as body size (Makvandi-Nejad et al. 2012; Petersen et al. 2013; Metzger et al. 2013), diseases (Sharav et al. 2017), reproduction (Metzger et al. 2015) as well as physical performance (Schröder et al. 2012; Moon et al. 2015) in different breeds. However, the recent molecular genetic studies on athletic performance is still present only a first step toward a better understanding of the genetic factors that may influence horse ability, thus continuing research is necessary.

This study aimed to screen the potential candidate genes involved in athletic performance, using the high depth of whole-genome sequence data from different breeds including HNV warmblood horses. The findings here help advance our knowledge of the biological mechanisms controlling economically important traits in sport horse breeding.

\section{Material and methods}

\section{Population genome resequencing}

Here, to avoid bias from unequal sample sizes, an equal number of individuals for each horse breeds was considered. Sequencing analysis was based on data from four different horse breeds, HNV $(n=4)$, Akhal-Teke (AKH) $(n=4)$, Franches-Montagnes $(\mathrm{FCH})(n=4)$ and Standardbred (STD) $(n=4)$ (Supplementary Table S1), were downloaded from the Sequence Read Archive (SRA) at NCBI (https://trace.ncbi.nlm.nih.gov/Traces/sra). Reference genome sequence of horse, Equus_caballusEquCab2, was downloaded from ENSEMBL (version 94) 
ftp://ftp.ensembl.org/pub/release-94/fasta/equus caballus/dna/. Raw sequence data were evaluated using fastQC (www.bioinformatics. babraham.ac.uk/projects/fastqc/). Low-quality bases in sequence reads were filtered using Btrim (Kong, 2011). For the cutoff quality score of trimming, we chose the $1 \%$ probability of error, which corresponded to a Phred quality score of 20 (Q20). The sequence reads shorter than 25 bp ( $>25$ bases), also were removed (Yun and Yun 2014). Qualified reads were aligned onto the references genome using BWA-MEM with default settings except for “-t 8 -M -R” options (bio-bwa.sourceforge.net) (Supplementary Table S2). We used open-source software packages for downstream processing and variant calling. PCR duplicated were marked and removed using Picard tools (http://picard.sourceforge.net, version 2.0.1). Genome Analysis Toolkit 3.7 (GATK) (www.broadinstitute.org/gatk) was used to perform local realignment of reads to correct misalignments due to the presence of indels ("RealignerTargetCreator" and "IndelRealigner" arguments). Variant calling and filter tagging were performed by UnifiedGenotyper and VariantFiltration command in GATK. After SNPs calling, all variants exhibited mapping quality $<25$ and genotype quality $<40$ were removed. And loci with more than two alleles and within clusters ( $>3$ SNPs in a 10-bp window) were discarded from further analysis. To determine SNPs that have protein-altering effects, all SNPs were assigned to specific genomic regions using ANNOVAR software (Wang et al. 2010) based on the Ensembl horse annotations.

\section{Population variation analysis}

To understand the genetic distance between the breeds considered, we constructed a neighbourjoining tree using the software megacc (v7.0.26-1) (Kumar et al. 2016) with 1000 bootstrap replications. With a pruned data set, which discarded SNPs contributed by regions of extensive 
strong linkage disequilibrium (LD) across breeds (Plink --indep 10050 0.1), we implemented a Bayesian model-based approach to view the population structure (Alexander et al. 2009) with an ancestor population size ranging from 2 to 4 . In addition, using the all autosomal SNPs, a genomic relatedness matrix (GRM) between pairwise individuals was estimated and principal component analysis (PCA) was performed by GCTA (Yang et al. 2011). Linkage disequilibrium (LD) and Runs of homozygosity (ROHs) were also estimated by the PLINK program 1.07 (Purcell et al. 2007).

\section{Genome-wide selective sweeps scan}

Two approaches were used to identify the genomic regions harboring footprints of positive selection in HNV horse. We compared the genome of HNV horse, used as a test population, with other three breeds (AKH, FCH and STB grouped into one population), used as a reference population. The fixation index (Fst) values for each SNP were estimated between two populations as described elsewhere (Akey et al. 2002). Sliding window analysis was performed with a window size of $50 \mathrm{~kb}$ and a step size of $25 \mathrm{~kb}$. In addition, cross-population composite likelihood ratio (XP-CLR) values for each variant were calculated with a script available at http://genetics.med.harvard.edu/reich/Reich_Lab/Software.html. Our parameters were as follows: sliding window size $0.1 \mathrm{cM}$, grid size $10 \mathrm{~kb}$, maximum number of SNPs within a window 300, correlation level for 2 SNPs weighted with a cutoff of 0.99 . Protein-coding genes at the regions with higher values of Fst and XP-CLR (top 1\%) were retrieved as candidate selective sweeps.

\section{Gene enrichment analysis}


We used the Variant Effect Predictor (http://asia.ensembl.org/info/docs/tools/index.html) and g:Profiler (http://biit.cs.ut.ee/gprofiler/) (Reimand et al. 2011) for gene enrichment analysis to further understand the biological functions and pathways of the selected genes.

\section{Results and discussion}

\section{Population genetic structure and differentiation}

We first used several quality control (QC) steps to assure SNP data quality. These filters included mapping quality, genotype quality and missing data per each individual. A total of $18,874,321$ SNPs were kept after these QC steps. To assess the phylogenic relationship among the horses, we constructed a phylogenic tree of all four breeds, HNV, STB AKH and FCH, based on the information of complete autosomal SNPs. As we expected, all individuals were grouped into four separate horse breeds (Figure 1A). Our phylogenetic pattern was also supported by a principle component analysis (PCA) (Figure 1B).

Consistent with the phylogenetic tree, the AKH breed, originating in Middle east, had the greatest distance from the other three groups in the PCA analysis. We also used one Bayesian model-based analyses to infer general patterns of admixture from genetic data (Figure 2A). A clear separation was observed between STB and other three groups if two and three ancestral populations $(K)$ were assumed, respectively. When $K=4$, HNV horses showed closer relationship with FCH group, that is in agreement with the phylogenetic tree results.

\section{Linkage disequilibrium decay and Runs of homozygosity}

We studied the patterns of LD, which can be informative for population demography. Initial mean $\mathrm{r}^{2}$ was dropped rapidly across all the genome groups with the same reduction, 
approximately 0.03 , by $5 \mathrm{~kb}$. The extent of long-range LD was the highest in the STB and AKH, respectively (Figure 2B). ROHs were also quantified to assess genetic relatedness among individuals and recent inbreeding. Figure $2 \mathrm{C}$ shows summary of ROH in two length categories (more than $100 \mathrm{~kb}$ and $1000 \mathrm{~kb}$, respectively) which were observed across all 31 autosomes. The $\mathrm{AKH}, \mathrm{HNV}$ and $\mathrm{FCH}$ breeds presented similar pattern values in all two categories while the STB breed was the population with the higher proportion of short and long $\mathrm{ROH}$.

\section{Candidate Genes under Positive Selection}

Success in show-jumping performance depends on an excellent combination of quick and strong muscle contraction, as well as balance and strength from approach to takeoff. Nearly all of the force for jumping come from the hind limbs (Lopez-Rivero and Letelier 2000). Hence, it is clear that the genes associated with power elite performance and limb health are grouped under the potential candidate genes for show-jumping performance (Schröder et al. 2011). Here, to determine whether directional selection might have occurred in the HNV horses, we examined two different parameters. We first evaluated the population differentiation of HNV from the other three breeds to search for loci that show evidence of positive selection, using the Fst statistic, with a sliding window analysis (window size, $50 \mathrm{~kb}$ and step length, $25 \mathrm{~kb}$ ). We also extracted the top SNPs using cutoffs of $0.1 \%$, and obtained 483 SNPs which were mapped to 299 protein-coding genes. Although, gene set enrichment analysis did not show categories associated with athletic performance (Supplementary Table S3), functional enrichment analysis of the list of candidate genes revealed one protein coding gene, actin alpha 1 gene (ACTA1) located on ECA1, which is expressed in skeletal muscle and is potentially involved in athletic performance in HNV (Supplementary Table S4) (Figure 3A and 4A). 
The product encoded by this gene, skeletal muscle $\alpha$-actin, is the principal actin isoforms in the sarcomeric thin filaments of adult skeletal muscle. This protein is member of the actin protein family which are involved in many critical cell functions such as cell motility and cell morphology and also is essential for normal muscle contraction (Craig and Padrón 2004; Laing et al. 2009). Furthermore, gene expression analysis has found high mRNA expression levels of ACTA1 gene in the equine skeletal muscle (McGivney et al. 2010). Previous studies have shown that ACTA1 mutations are associated with athletic-performance in Thoroughbred horse (Gu et al. 2009), muscle weakness in human (Laing et al. 2009) as well as growth traits in cattle (Tian et al. 2011). Also recently, Brard and Ricard (2014) reported a quantitative trait locus (QTL) (BIEC2_31196) associated with show-jumping performance close (4.94 Mb) to ACTA1 gene in French sport horses.

In addition, we applied an XP-CLR test to search for allelic differentiation that must have occurred very fast due to random drift (Chen et al. 2010). A total of 485 protein-coding genes with high XP-CLR values $(P<0.01)$ were identified (Supplementary Table S5). Gene set enrichment analysis of the 458 genes found some GO categories related with movement and development process, such as "Locomotion" (GO:0040011), "Cell development” (GO:0048468), "Regulation of multicellular organismal development" (GO:2000026), "Anatomical structure development" (GO:0048856) and "Developmental process" (GO:0032502) (Supplementary Table S6). Athletic performance is ultimately the result of the actions of muscles. In horses, the differences between the locomotion patterns, which show a noticeable variation between breeds, are extremely valuable traits (Andersson et al. 2012). Previous studies have demonstrated that the Hanoverians are bred to be willing and trainable, which have powerful body, athletic movement and strong limbs (Árpád, 2011). 
Here, we found $A C T A 1$ and other three protein coding genes (RYR2,IGF-IR and PPARD) that are potentially involved in athletic-performances (Figure 3B). Additionally, haplotype comparison analysis revealed a consistent differentiation of ACTAl for HNV horse from other horses (Figure 4B). RYR2 (encoding the cardiac ryanodine receptor type 2), is primarily expressed in the heart, which is the major $\mathrm{Ca}^{++}$release channel in cardiac muscle (Priori et al. 2001; Lanner et al. 2010). It has been reported that RYR2 gene is close $(0.55 \mathrm{Mb})$ to one QTL region associated with jumping performance in French sport horses (Brard and Ricard 2014). $I G F-1$ is a pleiotropic growth factor that plays a central role in the regulation of cell proliferation, survival and differentiation as well as is a key factor in regulating skeletal muscle and development. The biological function of $I G F-1$ is mediated by the IGF-1 receptor (IGF-1R) (LeRoith 1997; Delafontaine et al. 2004; Yakar et al. 2010). It has been claimed that the global deletion of $I G F-1 R$ from chondrocyte in the long bones leads to both skeletal defects and cartilage changes (Yakar et al. 2010). Previous studies have also found that the $I G F-1 R$ mutations are associated with several growth traits in cattle (Szewczuk et al. 2013), chicken (Lei et al. 2008) as well as total size in dog (Hoopes et al. 2012). Another physical performancerelated gene, peroxisome proliferator activated receptor delta (PPARD), was found in the selection regions in ECA20. This gene encodes peroxisome proliferator-activated receptor delta, which regulates the expression of suites of genes involved in carbohydrate and lipid metabolism (Barbier et al. 2002). The expression levels of this transcriptional co-activator is increased by fasting and physical exercise, thus triggering adaptation to physical exercise in the skeletal muscle (de Lange et al. 2008). A previous study indicated that a mutation (rs2016520) on PPARD gene had potentially effect on physical performance in human (Eynon et al. 2009). 
In summary, using whole genome comparative analysis, we identified ACTAI and other three candidate genes (RYR2, IGF-1R and PPARD) in Hanoverian horses. These genes may have been under positive selection due to their essential roles in Hanoverians athletic performance. Our findings will facilitate future genome-wide association studies and investigations into genomic targets of selection for such traits in horse breeding. However, further investigation is required to validate these candidate genes in larger data sets and with multiple horse populations.

\section{Supplementary Material}

Supplementary data are available at Genome online.

\section{Conflict of Interest}

The authors declare no conflict of interests. 


\section{References}

Akey JM, Zhang G, Zhang K, Jin L, Shriver MD. 2002. Interrogating a high-density SNP map for signatures of natural selection. Genome Research 12, 1805-1814. https://doi.org/10.1101/gr.631202

Alexander DH, Novembre J, Lange K. 2009. Fast model-based estimation of ancestry in unrelated individuals. Genome Research 19, 1655-1664. https://doi.org/10.1101/gr.094052.109

Andersson LS, Larhammar M, Memic F et al. 2012. Mutations in DMRT3 affect locomotion in horses and spinal circuit function in mice. Nature 488, 642-646. doi: https://doi.org/10.1038/nature11399

Árpád B. 2011. Sport horse breeding. Kaposvar University. https://www.tankonyvtar.hu.

Barbier O, Torra JP, Duguay Y, Blanquart C, Fruchart JC, Glineur C, Staels B. 2002. Pleiotropic actions of peroxisome proliferator-activated receptors in lipid metabolism and atherosclerosis. Arteriosclerosis, Thrombosis, and Vascular Biology 22, 716-726. https://doi.org/10.1161/01

Brard S, Ricard A. 2014. Genome-wide association study for jumping performances in French sport horses. Animal Genetics 46, 78-81. https://doi.org/10.1111/age.12245

Chen H, Patterson N, Reich D. 2010. Population differentiation as a test for selective sweeps. Genome Research 20, 393-402. https://doi.org/10.1101/gr.100545.109

Craig RW, Padrón R. 2004. Molecular structure of the sarcomere. In: Engel AG, FranziniArmstrong LC, editors. Myology, Basic and Clinical. New York: McGraw-Hill. pp. 129166. 
Delafontaine P, Song YH, Li Y. 2004. Expression, Regulation, and Function of IGF-1, IGF-1R, and IGF-1 Binding Proteins in Blood Vessels. Arteriosclerosis, Thrombosis, and Vascular Biology 24, 435-444.

De Lange P, Lombardi A, Silvestri E, Goglia F, Antonia Lanni A, Moreno M. 2008. Peroxisome Proliferator-Activated Receptor Delta: A Conserved Director of Lipid Homeostasis through Regulation of the Oxidative Capacity of Muscle. PPAR Research 172676, 7. doi: $10.1155 / 2008 / 172676$

Eynon N, Meckel Y, Alves AJ, Yamin C, Sagiv M, Goldhammer E, Sagiv M. 2009. Is there an interaction between PPARD T294C and PPARGC1A Gly482Ser polymorphisms and human endurance performance?. Exp Physiology 94, 1147-1152.

https://doi.org/10.1113/expphysiol.2009.049668

Gu J, Orr N, Park SD, Katz LM, Sulimova G, MacHugh DE, Hill EW. 2009. A genome scan for positive selection in thoroughbred horses. PLoS One 4, e5767. https://doi.org/10.1371/journal.pone.0005767

Hamann H. Distl O. 2008. Genetic variability in Hanoverian warmblood horses using pedigree analysis. Journal of Animal Science 86, 1503-1513. https://doi.org/10.2527/jas.2007-0382.

Hoopes BC, Rimbault M, Liebers D, Ostrander EA, Sutter NB. 2012. The insulin-like growth factor 1 receptor (IGF1R) contributes to reduced size in dogs. Mammalian Genome 23, 78090. https://doi.org/10.1007/s00335-012-9417-z.

Koenen EPC, Aldridge LI, Philipsson J. 2004. An overview of breeding objectives for warmblood sport horses. Livestock Production Science 88, 77-84. https://doi.org/10.1016/j.livprodsci.2003.10.011 
Kong Y. 2011. Btrim: a fast, lightweight adapter and quality trimming program for nextgeneration sequencing technologies. Genomics 98, 152-153 https://doi: 10.1016/j.ygeno.2011.05.009

Kumar S, Stecher G, Tamura K. 2016. MEGA7: molecular evolutionary genetics analysis version 7.0 for bigger datasets. Molecular Biology and Evolution 33, 1870-1874. https://doi.org/10.1093/molbev/msw054

Laing NG, Dye DE, Wallgren-Pettersson C, Richard G, Monnier N, Lillis S, Winder TL, Lochmüller H, Graziano C, Mitrani-Rosenbaum S, Twomey D, Sparrow JC,. Beggs AH, Nowak KJ. 2009. Mutations and polymorphisms of the skeletal muscle alpha-actin gene (ACTA1). Human Mutation 30, 1267-77. https://doi.org/10.1002/humu.21059

Lanner JT, Georgiou DK, Joshi AD, Hamilton, SL. 2010. Ryanodine Receptors: Structure, Expression, Molecular Details, and Function in Calcium Release. Cold Spring Harbor perspectives in biology 2, a003996. https://doi.org/10.1101/cshperspect.a003996

Lei M, Peng X, Zhou M, Luo C, Nie Q, Zhang X. 2008. Polymorphisms of the IGF1R gene and their genetic effects on chicken early growth and carcass traits. BMC Genetics 9, 70. https://doi.org/10.1186/1471-2156-9-70.

LeRoith D. 1997. Seminars in medicine of the Beth Israel Deaconess Medical Center. Insulinlike growth factors. New England Journal of Medicine 336, 633-640.

Leroy H, Rowen L. 2013. The Human Genome Project: big science transforms biology and medicine. Genome Medicien 5, 79. https://doi.org/ 10.1186/gm483

Lopez-Rivero JL, Letelie, A. 2000. Skeletal muscle profile of show jumpers: physiological and pathological considerations. The elite show jumper. Conference of Equine Sports Medicine Science 57-76. 
Makvandi-Nejad S, Hoffman GE, Allen JJ, Chu E, Gu E, Chandler AM, Loredo AI, Bellone RR, Mezey JG, Brooks SA, Sutter NB. 2012. Four Loci Explain 83\% of Size Variation in the Horse. PLoS ONE 7, e39929. https://doi.org/10.1371/journal.pone.0039929

McGivney BA et al. 2010. Characterization of the equine skeletal muscle transcriptome identifies novel functional responses to exercise training. BMC Genomics 11, 398. https://doi.org/10.1186/1471-2164-11-398

Metzger J, Karwath M, Tonda R, Beltran S, Águeda L, Gut M, Gut IG, Distl O. 2015. Runs of homozygosity reveal signatures of positive selection for reproduction traits in breed and non-breed horses. BMC Genomics 16, 764. https://doi.org/10.1186/s12864-015-1977-3

Metzger J, Schrimpf R, Philipp U, Distl O. 2013. Expression levels of LCORL are associated with body size in horses. Plos One 8, e56497. https://doi.org/10.1371/journal.pone.0056497

Moon S, Lee JW, Shin D, Shin KY, Kim J, Choi LY, Kim J, Kim H. 2015. A Genome-wide Scan for Selective Sweeps in Racing Horses. Asian-Australas Jouranl of Animal Science 28, 1525-1531. https://doi.org/10.5713/ajas.14.0696

Moradi MH, Nejati-Javaremi A, Moradi-Shahrbabak M, Dodds K, Mcewan J. 2012. Genomic scan of selective sweeps in thin and fat tail sheep breeds for identifying of candidate regions associated with fat deposition. BMC Genetics 13, 10. https://doi.org/10.1186/1471-2156-1310.

Petersen JL, Mickelson JR, Cothran EG, Andersson LS, Axelsson J. et al. 2013. Genetic Diversity in the Modern Horse Illustrated from Genome-Wide SNP Data. Plos One 8, e29013. https://doi.org/10.1371/journal.pone.0054997 
Priori SG, Napolitano C, Tiso N, Memmi M, Vignati G. et al. 2001. Mutations in the cardiac ryanodine receptor gene (hRyR2) underlie catecholaminergic polymorphic ventricular tachycardia. Circulation 16, 196-200. https://doi.org/ 10.1161/01.CIR.103.2.196

Purcell S. et al. 2007. PLINK: a tool set for whole-genome association and population-based linkage analyses. American Society of Human Genetics 81, 559-575. https://doi.org/10.1086/519795

Reimand J, Arak T, Vilo J. 2011. g: Profiler--a web server for functional interpretation of gene lists (2011 update). Nucleic Acids Research 39, W307-315. https://doi.org/10.1093/nar/gkr378

Rothammer S, Seichter D, Forster M, Medugorac I. 2013. A genome-wide scan for signatures of differential artificial selection in ten cattle breeds. BMC Genomics 14, 908. https://doi.org/10.1186/1471-2164-14-908

Schröder W, Klostermann A, Distl O. 2011. Candidate genes for physical performance in the horse. Veterinary Journal 190, 39-48. https://doi.org/10.1016/j.tvj1.2010.09.029

Schröder W, Klostermann A, Stock KF, Distl O. 2012. A genome-wide association study for quantitative trait loci of show-jumping in Hanoverian warmblood horses. Animal Genetics 43, 392-400. https://doi.org/10.1111/j.1365-2052.2011.02265.x

Sharav T, Satoru Konnai S, Ochirkhuu N, Ochir ETS, Mekata H, Sakoda Y, Umemura T, Murata S, Chultemdorj T, Ohashi K. 2017. Detection and molecular characterization of equine infectious anemia virus in Mongolian horses. Journal of Veterinary Medical Science 79, 1884-1888. https://doi.org/10.1292/jvms.17-0202

Szewczuk M, Zych S, Wójcik J, Czerniawska-Piątkowska E. 2013. Association of two SNPs in the coding region of the insulin-like growth factor 1 receptor (IGF1R) gene with growth- 
related traits in Angus cattle. Journal of Applied Genetics 54, 305-8. https://doi.org/10.1007/s13353-013-0155-z

Tian W, Zhang S, Li X, Gao Q, Jin X, Yan C. 2011. Genetic polymorphism and correlation analysis with growth traits of ACTA1 gene in Yanbian Yellow cattle. China Animal Husbandry \& Veterinary Medicine 38, 153-157.

Tomasz Próchniak T, Rozempolska-Rucińska I, Zięba G, Marek Łukaszewicz M. 2015. Genetic Variability of Show Jumping Attributes in Young Horses Commencing Competing. AsianAustralas Journal of Animal Science 28, 1090-1094. https://doi.org/10.5713/ajas.14.0866

Wang K, Li MY, Hakonarson H. 2010. ANNOVAR: functional annotation of genetic variants from high-throughput sequencing data. Nucleic Acids Research 38, 16. https://doi.org/10.1093/nar/gkq603

Wilkinson, S et al. 2013. Signatures of diversifying selection in European pig breeds. PLoS Genet 9, e1003453. https://doi.org/10.1371/journal.pgen.1003453

Yakar S, Courtland H-W, Clemmons D. 2010. IGF-1 and bone: New discoveries from mouse models. Journal of Bone and Mineral Research 25, 2543-2552. https://doi.org/10.1002/jbmr.234.

Yamasaki M, Tenaillon MI, Bi IV, Schroeder SG, Sanchez-Villeda H, Doebley JF, Gaut BS, McMullen MD. 2005. A large-scale screen for artificial selection in maize identifies candidate agronomic loci for domestication and crop improvement. Plant Cell 17, 2859-72. https://doi.org/10.1105/tpc.105.037242

Yang J et al. 2011. GCTA: a tool for genome-wide complex trait analysis. American Journal of Human Genetic 88, 76-82. https://doi.org/10.1016/j.ajhg.2010.11.011 
Yun S, Yun S. 2014. Masking as an effective quality control method for next-generation sequencing data analysis. BMC Bioinformatics 15 (1): 382. https://doi.org/10.1186/s12859$\underline{014-0382-2}$

Zhang DX, Hewitt GM. 2003. Nuclear DNA analyses in genetic studies of populations: practice, problems and prospects. Molecular Ecology 12, 563-584. https://doi.org/10.1046/j.1365294X.2003.01773.x 


\section{Legends to Figures}

Figure. 1 Phylogenic Analysis of horse breeds. (A) Phylogenic tree constructed based on the neighbour-joining tree method with 1000 bootstrap replications. (B) Principle component Analysis. HNV: Hanoverian, AKH: Akhal-Teke, FCH: Franches-Montagnes, STD: Standardbred Figure. 2 Analysis of population variation. (A) Population structure; $K=2$ is the best. (B) LD decay. (C) Runs of Homozygosity (ROH). HNV: Hanoverian, AKH: Akhal-Teke, FCH: Franches-Montagnes, STD: Standardbred

Figure. 3 Genomic landscape of population differentiation by $F_{S T}(A)$ and XP-CLR (B) Figure. 4 Population differentiation in chr1:68.00-Mb-68:74 between Hanoverian and other horses. (A) Landscape of Fst. (B) Haplotype comparison between Hanoverian and other horses

\section{Supporting information}

Additional supporting information may be found online in the supporting information tables for this article:

Table S1. Sample information for each horse used in this study.

Table S2. Genome sequence coverage and mapping information for all horses in this study.

Table S3. Overrepresented GO categories among genes showing high Fst values in HNV.

Table S4. Positively selected genes identified by Fst method.

Table S5. Positively selected genes identified by XP-CLR method.

Table S6. Gene functional enrichment categories showing high XP-CLR values in HNV. 
A

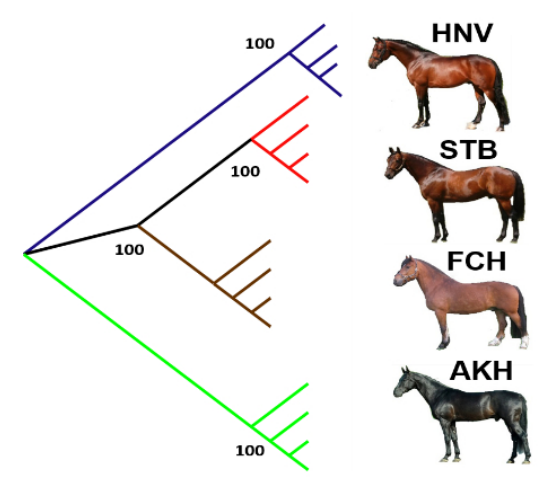

B

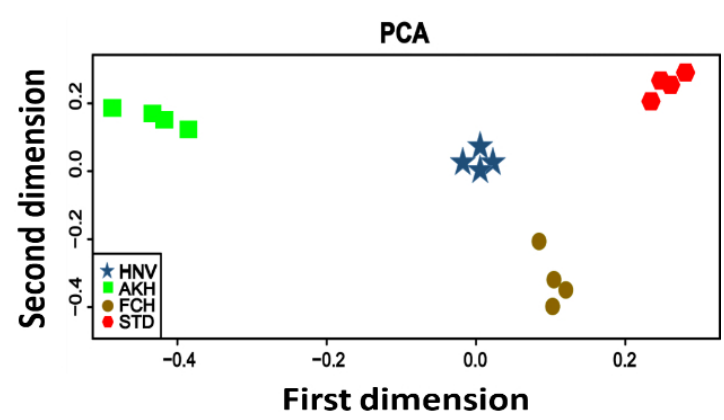

Figure. 1 Phylogenic Analysis of horse breeds. (A) Phylogenic tree constructed based on the neighbourjoining tree method with 1000 bootstrap replications. (B) Principle component Analysis. HNV: Hanoverian, AKH: Akhal-Teke, FCH: Franches-Montagnes, STD: Standardbred 


\section{A $\mathrm{K}=2 \quad \mathrm{~K}=3 \quad \mathrm{~K}=4$}
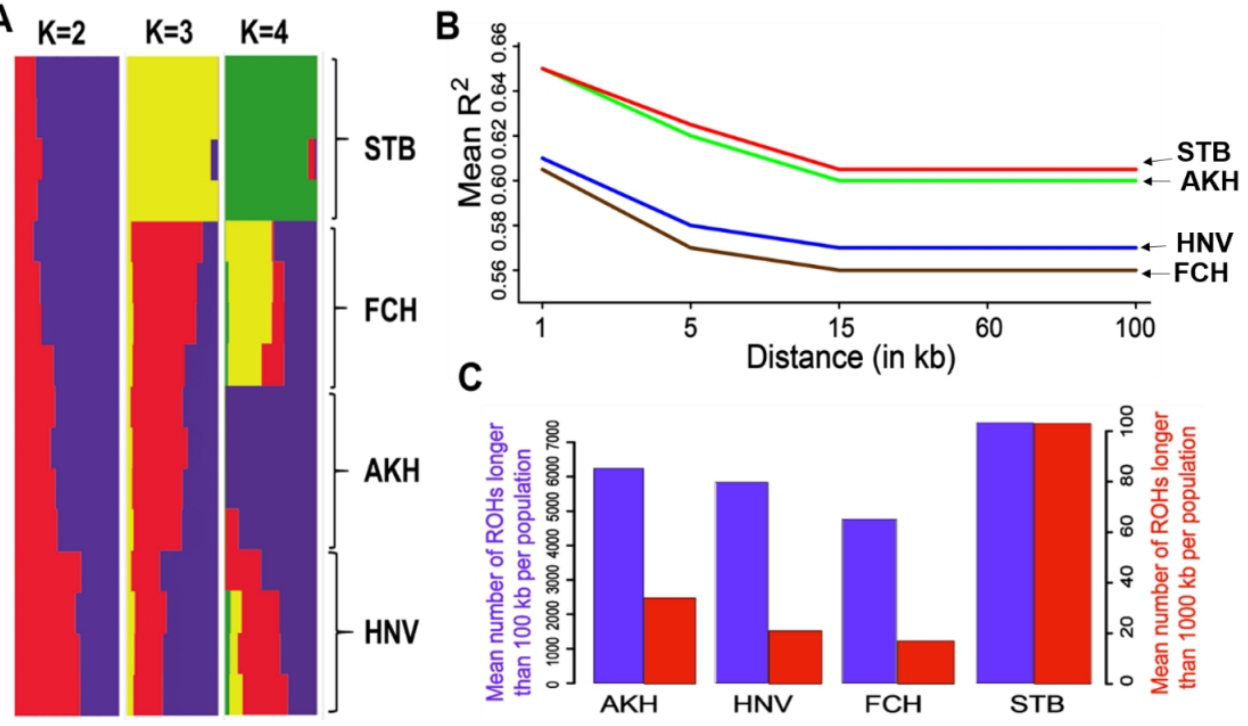

C
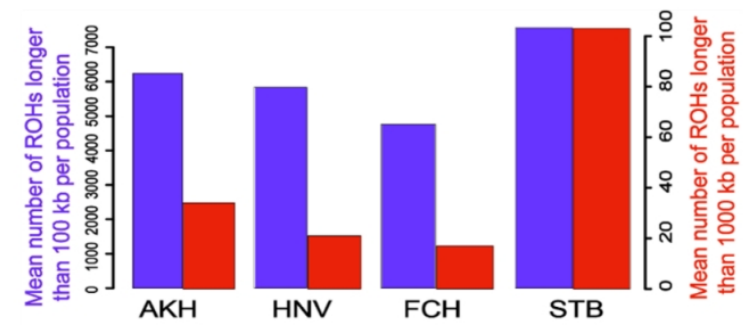

Figure. 2 Analysis of population variation. (A) Population structure; $K=2$ is the best. (B) LD decay. (C) Runs of Homozygosity (ROH). HNV: Hanoverian, AKH: Akhal-Teke, FCH: Franches-Montagnes, STD: Standardbred 


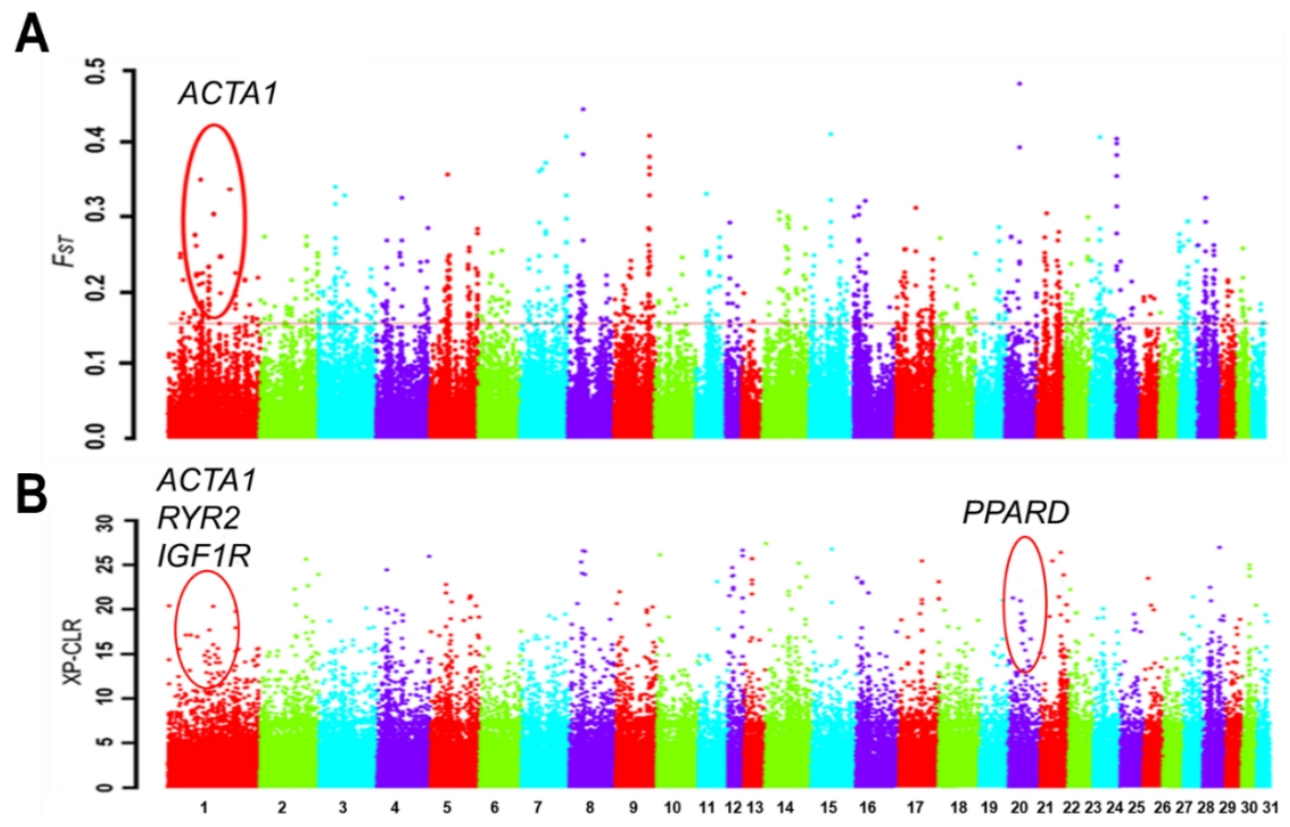

Figure. 3 Genomic landscape of population differentiation by FST (A) and XP-CLR (B) 
A

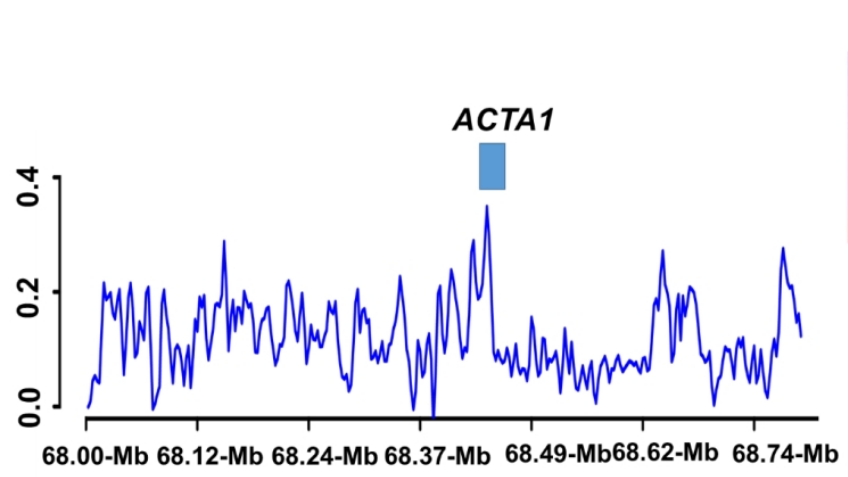

B

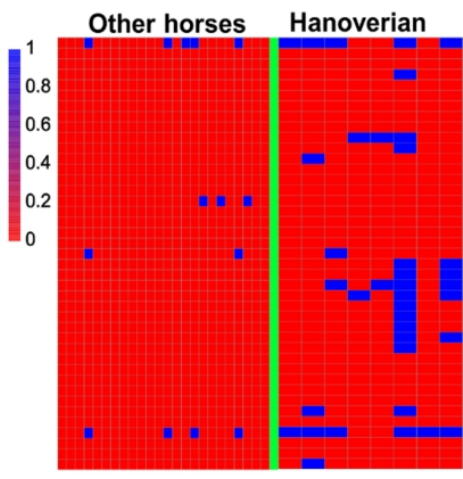

1:68408852-68411553

Figure. 4 Population differentiation in chr1:68.00-Mb-68:74 between Hanoverian and other horses. (A) Landscape of Fst. (B) Haplotype comparison between Hanoverian and other horses 